$\xi=-\mathbf{Z}^{2}$

\title{
Characteristics exponents of the triangular solution in the elliptical restricted three-body problem under radiating and triaxial primaries
}

\author{
A. Narayan ${ }^{1 *}$, Krishna Kumar Pandey ${ }^{2}$, Sandip Kumar Shrivastava ${ }^{2}$ \\ ${ }^{1}$ Department of Mathematics, Bhilai Institute of Technology, Durg, 491001, India \\ ${ }^{2}$ Department of Mathematics, ShriShankaracharya Institute of Technology and Management,Bhilai,490020 \\ *Corresponding author E-mail:ashutoshmaths.narayan@gmail.com
}

\begin{abstract}
This Paper deals with the effects of the radiation pressure and triaxiality of primaries on the stability of infinitesimal motion about triangular equilibrium points $\left[L_{4}, L_{5}\right]$ in the elliptical restricted three body problem (ER3EB) around binary system. For determining the characteristic exponents of variational equations with periodic coefficient, we have used analytical method, described by Bennet in [3, 4]. This analytical method is based on Floquet's theory. The stability of equilibrium points has been discussed under the assumption thatboth the primaries are radiating and triaxial. For this we have drawn transition cureves in $\mu$-e plane. And it is seen that system is stable outside the transition curves, while system is Unstable within the transition curves.
\end{abstract}

Keywords:ER3BP; Dynamical System Libration Points; Flouqet Theory.

\section{Introduction}

The restricted three- body problem has a wider range of application that the general three body problem in space dynamics, celestial mechanics and analytical dynamics. The elliptical restricted three body problem (ER3BP) models the motion of infinitesimal mass which moves under the influence of two massive bodies revolving around their centre of mass in an elliptical orbit, being influenced but not influencing the two primaries. The circular restricted three body problem has been generalized by the introduction of the elliptic orbit, thus improving its applicability and retaining some useful properties of the circular model suitable to the elliptic case. Various authors and researchers like Ammar [1] ,Grebnikov [7],Gyorgyey [8], Kumar, S., Ishwar B [9], Kumar, V., Choudhary , R. K. [10] , V. V., Perdios, E., Labrapoulou, P.[11], Moulton, F.R.[12], Narayan, A., Kumar, C.R.[13], Narayan, A., Singh, N.[14], Narayan, A., Usha, T.[15] , Singh, J., Aishetu, U. (2012), Singh, J., Aishetu, U.[18], Szebebely, V .[20],Usha, T., Narayan, A., Ishwar, B. [21],Zimvoschikov, A.S., Thkai, V.N.[22] , have studied the effects of radiation pressure on the motion of the infinitesimal body by taking one or both primaries as a source of radiation.

Danby [5] examined the stability of the infinitesimal motion about the triangular points in the ER3BP; using a digital computer numerical scheme based on Floquet theory giving the result in the form of transition curves in $\mu-$ e plane. Bennet $[3,4]$ further studied the problem by taking the characteristics exponents of the five equilibrium points in elliptical restricted problem. This method is essentially the same as employed by Moulton, F.R.[12] in his classical study of eight satellite of Jupiter.Alfriend, K.T., Rand, R. $\mathrm{H}$. [2] investigated the same problem using two variable expansion methods and verified the result of Danby [5]. Further,Deprit, A., Rom, A. [6] used the perturbation method based on Lie transform to develop the characteristics exponents of the monodromy matrix in power series of small parameter. They produced the principal parts of the characteristics exponents as general functions of mass ratio.

The present study is devoted to the analysis of the stability of triangular points under radiating and triaxial primaries by exploiting the analytical technique developed by Bennet $[3,4]$. This method is based on Floquet's theory for the determination of characteristics exponents for a system with periodic coefficients. The transition curves have been presented through the simulation technique, which shows the region of stability as well as instability for different values of radiation pressures and triaxial parameters.

\section{Variational equation of motion}

The differential equations of motion of the infinitesimal mass in the elliptical restricted three body problem (ER3BP) under radiating and triaxial primaries in a barycentric, pulsating system are given as: Narayan,A., Pandey, K.K and Shrivastava,S.K. [17].

The differential equation of motion of the third body $\mathrm{P}$ in nondimensional barycentre, pulsating and non-uniformly rotating coordinate system $(\mathrm{x}, \mathrm{y})$ is written in the form:

$$
\begin{aligned}
& \mathrm{x}^{\prime \prime}-2 \mathrm{y}^{\prime}=\frac{1}{1+\mathrm{ecos} v}\left(\frac{\partial \Omega}{\partial \mathrm{x}}\right) \\
& \mathrm{y}^{\prime \prime}+2 \mathrm{x}^{\prime}=\frac{1}{1+\mathrm{e} \cos v}\left(\frac{\partial \Omega}{\partial \mathrm{y}}\right)
\end{aligned}
$$

Where ' denotes differentiation with respect to $v$, and

$$
\begin{aligned}
& \Omega=\frac{\mathrm{x}^{2}+\mathrm{y}^{2}}{2}+\frac{1}{\mathrm{n}^{2}}\left[\frac{(1-\mu) \mathrm{q}_{1}}{\mathrm{r}_{1}}+\frac{\mu \mathrm{q}_{2}}{\mathrm{r}_{2}}+\frac{(1-\mu)\left(2 \sigma_{1}-\sigma_{2}\right) \mathrm{q}_{1}}{2 \mathrm{r}_{1}^{3}}+\frac{\mu\left(2 \sigma_{1}{ }^{\prime}-\sigma_{2}{ }^{\prime}\right) \mathrm{q}_{2}}{2 \mathrm{r}_{2}^{3}}-\right. \\
& \left.\frac{3(1-\mu)\left(\sigma_{1}-\sigma_{2}\right) \mathrm{y}^{2} \mathrm{q}_{1}}{2 \mathrm{r}_{1}^{5}}-\frac{3 \mu\left(\sigma_{1}{ }^{\prime}-\sigma_{2}{ }^{\prime}\right) \mathrm{y}^{2} \mathrm{q}_{2}}{2 \mathrm{r}_{2}^{5}}\right] \\
& \text { Where }
\end{aligned}
$$




$$
\begin{aligned}
& \mathrm{n}^{2}=1+\frac{3}{2}\left(2 \sigma_{1}-\sigma_{2}\right)+\frac{3}{2}\left(2 \sigma_{1}^{\prime}-\sigma_{2}^{\prime}\right)(2.3) \\
& \mathrm{r}_{1}^{2}=(\mathrm{x}+\mu)^{2}+\mathrm{y}^{2} \\
& \mathrm{r}_{2}^{2}=(\mathrm{x}+\mu-1)^{2}+\mathrm{y}^{2}
\end{aligned}
$$

Where

$$
\mu=\frac{m_{2}}{m_{1}+m_{2}}
$$

Where $m_{1}$ and $m_{2}$ are masses of the primaries. $q_{1}, q_{2}$ are the radiation pressure. $\sigma_{1}, \sigma_{2}, \sigma_{1}^{\prime}$ and $\sigma_{2}^{\prime}$ are triaxial parameters, while $\mathrm{e}$ and $v$ are the eccentricity of orbits and true anomaly of the primaries respectively.

The coordinates of the triangular points $\mathrm{L}_{4}$ and $\mathrm{L}_{5}$ are given by Narayan, A., Pandey, K.K and Shrivastava,S.K. [17].

$\mathrm{x}=\frac{1}{2}-\mu+\frac{\left(\epsilon_{2}^{\prime}-\epsilon_{1}^{\prime}\right)}{3}+\left[\frac{-3}{8}-\epsilon_{1}^{\prime}-\frac{(1-\mu)}{2 \mu}+\frac{(1-\mu) \epsilon_{1}^{\prime}}{2 \mu}\right] \sigma_{1}+$

$\left[\frac{7}{8}+\frac{\epsilon_{1}^{\prime}}{2}+\frac{(1-\mu)}{2 \mu}-\frac{(1-\mu) \epsilon_{1}^{\prime}}{2 \mu}\right] \sigma_{2}+\left[\frac{3}{8}-\frac{3 \mu}{8(1-\mu)}+\frac{3 \mu \epsilon_{2}^{\prime}}{8(1-\mu)}+\epsilon_{2}^{\prime}\right] \sigma_{1}{ }^{\prime}+$

$\left[\frac{-7}{8}+\frac{7 \mu}{8(1-\mu)}-\frac{7 \mu \epsilon_{2}^{\prime}}{8(1-\mu)}-\frac{\epsilon_{2}^{\prime}}{2}\right] \sigma_{2}^{\prime}$

$\mathrm{y}= \pm \frac{\sqrt{3}}{2}\left[1+\frac{2}{3}\left\{-\frac{\left(\epsilon_{1}^{\prime}+\epsilon_{2}^{\prime}\right)}{3}+\left[\frac{-19}{8}-\epsilon_{1}^{\prime}+\frac{(1-\mu)}{2 \mu}-\frac{(1-\mu) \epsilon_{1}^{\prime}}{2 \mu}\right] \sigma_{1}+\right.\right.$

$\left[\frac{15}{8}+\frac{\epsilon_{1}^{\prime}}{2}-\frac{(1-\mu)}{2 \mu}+\frac{(1-\mu) \epsilon_{1}^{\prime}}{2 \mu}\right] \sigma_{2}+\left[\frac{-19}{8}-\frac{3 \mu}{8(1-\mu)}+\frac{3 \mu \epsilon_{2}^{\prime}}{8(1-\mu)}-\epsilon_{2}^{\prime}\right] \sigma_{1}{ }^{\prime}+$

$\left.\left.\left[\frac{15}{8}+\frac{7 \mu}{8(1-\mu)}-\frac{7 \mu \epsilon_{2}^{\prime}}{8(1-\mu)}+\frac{\epsilon_{2}^{\prime}}{2}\right] \sigma_{2}^{\prime}\right\}\right]$

Now the equation of motion around the equilibrium points $\mathrm{L}_{4}$ are given by

$\xi^{\prime \prime}-2 \eta^{\prime}=\varnothing\left[\xi \Omega_{\mathrm{xx}}^{0}+\eta \Omega_{\mathrm{xy}}^{0}\right]$

$\eta^{\prime \prime}+2 \xi^{\prime}=\varnothing\left[\xi \Omega_{\mathrm{yx}}^{0}+\eta \Omega_{\mathrm{yy}}^{0}\right]$

Where $\xi, \eta$ denote small displacement in $\left(\mathrm{x}_{0}, \mathrm{y}_{0}\right)$.

Then $x=x_{0}+\xi, y=y_{0}+\eta$

Differentiating we get:

$\mathrm{x}^{\prime}=\xi^{\prime}, \mathrm{y}^{\prime}=\eta^{\prime}$

And

$\mathrm{x}^{\prime \prime}=\xi^{\prime \prime}, \mathrm{y}^{\prime \prime}=\eta^{\prime \prime}$

Where

$\emptyset=\left[\frac{1}{1+\mathrm{ecos} v}\right]$

The coordinates of equilibrium points are given by

$\mathrm{X}_{0}=\frac{1}{2}-\mu+\frac{\left(\epsilon_{2}^{\prime}-\epsilon_{1}^{\prime}\right)}{3}+\left[\frac{-3}{8}-\epsilon_{1}^{\prime}-\frac{(1-\mu)}{2 \mu}+\frac{(1-\mu) \epsilon_{1}^{\prime}}{2 \mu}\right] \sigma_{1}+$

$\left[\frac{7}{8}+\frac{\epsilon_{1}^{\prime}}{2}+\frac{(1-\mu)}{2 \mu}-\frac{(1-\mu) \epsilon_{1}^{\prime}}{2 \mu}\right] \sigma_{2}+\left[\frac{3}{8}-\frac{3 \mu}{8(1-\mu)}+\frac{3 \mu \epsilon_{2}^{\prime}}{8(1-\mu)}+\epsilon_{2}^{\prime}\right] \sigma_{1}{ }^{\prime}+$

$\left[\frac{-7}{8}+\frac{7 \mu}{8(1-\mu)}-\frac{7 \mu \epsilon_{2}^{\prime}}{8(1-\mu)}-\frac{\epsilon_{2}^{\prime}}{2}\right] \sigma_{2}^{\prime}$

$\mathrm{y}_{0}= \pm \frac{\sqrt{3}}{2}\left[1+\frac{2}{3}\left\{-\frac{\left(\epsilon_{1}^{\prime}+\epsilon_{2}^{\prime}\right)}{3}+\left[\frac{-19}{8}-\epsilon_{1}^{\prime}+\frac{(1-\mu)}{2 \mu}-\frac{(1-\mu) \epsilon_{1}^{\prime}}{2 \mu}\right] \sigma_{1}+\right.\right.$ $\left[\frac{15}{8}+\frac{\epsilon_{1}^{\prime}}{2}-\frac{(1-\mu)}{2 \mu}+\frac{(1-\mu) \epsilon_{1}^{\prime}}{2 \mu}\right] \sigma_{2}+\left[\frac{-19}{8}-\frac{3 \mu}{8(1-\mu)}+\frac{3 \mu \epsilon_{2}^{\prime}}{8(1-\mu)}-\epsilon_{2}^{\prime}\right] \sigma_{1}{ }^{\prime}+$ $\left.\left.\left[\frac{15}{8}+\frac{7 \mu}{8(1-\mu)}-\frac{7 \mu \epsilon_{2}^{\prime}}{8(1-\mu)}+\frac{\epsilon_{2}^{\prime}}{2}\right] \sigma_{2}^{\prime}\right\}\right]$

Now, differentiating $\Omega$ partially with respect to $\mathrm{x}, \mathrm{y}$ respectively and evaluating $\Omega_{\mathrm{xx}}, \Omega_{\mathrm{xy}}$ and $\Omega_{\mathrm{yy}}$ at the equilibrium points $\left(\mathrm{x}_{0}, \mathrm{y}_{0}\right)$.
$\Omega_{\mathrm{xx}}^{0}=\frac{3}{4}+\frac{\epsilon_{2}^{\prime}}{2}-\frac{3 \mu \epsilon_{1}^{\prime}}{2}-\frac{3 \mu \epsilon_{2}^{\prime}}{2}+\sigma_{1}\left[\frac{-3}{8}+\frac{69 \mu}{16}+\frac{169 \epsilon_{1}^{\prime}}{32}-\frac{39 \mu \epsilon_{1}^{\prime}}{32}-\frac{3}{4 \mu}+\right.$

$\left.\frac{7 \epsilon_{1}^{\prime}}{4 \mu}\right]+\sigma_{2}\left[\frac{15}{8}-\frac{75 \mu}{16}-\frac{625 \epsilon_{1}^{\prime}}{32}+\frac{599 \mu \epsilon_{1}^{\prime}}{32}+\frac{3}{4 \mu}-\frac{7 \epsilon_{1}^{\prime}}{4 \mu}\right]+\sigma_{1}^{\prime}\left[\frac{33}{16}-\right.$

$\left.\frac{147 \mu}{32}+\frac{3 \epsilon_{2}^{\prime}}{16}+\frac{191 \mu \epsilon_{2}^{\prime}}{16}+\frac{9 \mu^{2}}{16(1-\mu)}-\frac{15 \mu^{2} \epsilon_{2}^{\prime}}{16(1-\mu)}\right]+\sigma_{2}^{\prime}\left[\frac{-33}{16}+\frac{207 \mu}{32}-\right.$

$\left.\frac{7 \epsilon_{2}^{\prime}}{16}-\frac{483 \mu \epsilon_{2}^{\prime}}{32}-\frac{21 \mu^{2}}{16(1-\mu)}+\frac{35 \mu^{2} \epsilon_{2}^{\prime}}{16(1-\mu)}\right]$

$\Omega_{\mathrm{xy}}^{0}=\left\{\left(\frac{1}{2}-\mu-\frac{\epsilon_{1}^{\prime}}{9}-\frac{\mu \epsilon_{1}^{\prime}}{9}+\frac{2 \epsilon_{2}^{\prime}}{9}-\frac{14 \mu \epsilon_{2}^{\prime}}{9}\right)+\sigma_{1}\left[\frac{11}{24}-\frac{17 \mu}{24}+\right.\right.$

$\left.\frac{199 \epsilon_{1}^{\prime}}{48}-\frac{143 \mu \epsilon_{1}^{\prime}}{48}-\frac{1}{3 \mu}+\frac{17 \epsilon_{1}^{\prime}}{18 \mu}\right]+\sigma_{2}\left[\frac{3}{8}+\frac{13 \mu}{16}-\frac{557 \epsilon_{1}^{\prime}}{144}+\frac{445 \mu \epsilon_{1}^{\prime}}{144}+\frac{1}{3 \mu}-\right.$

$\left.\frac{17 \epsilon_{1}^{\prime}}{18 \mu}\right]+\sigma_{1}^{\prime}\left[\frac{7}{12}+\frac{31 \mu}{48}-\frac{161 \mu \epsilon_{2}^{\prime}}{36}-\frac{7 \epsilon_{2}^{\prime}}{6}-\frac{\mu^{2}}{4(1-\mu)}-\frac{\mu^{2} \epsilon_{2}^{\prime}}{12(1-\mu)}\right]+$

$\left.\sigma_{2}^{\prime}\left[\frac{-3}{4}-\frac{91 \mu}{48}+\frac{661 \mu \epsilon_{2}^{\prime}}{72}+\frac{47 \epsilon_{2}^{\prime}}{72}+\frac{7 \mu^{2}}{12(1-\mu)}+\frac{7 \mu^{2} \epsilon_{2}^{\prime}}{36(1-\mu)}\right]\right\}$

$\Omega_{\mathrm{yy}}^{0}=\frac{9}{4}+\frac{\epsilon_{1}^{\prime}}{2}-\epsilon_{2}^{\prime}-\frac{3 \mu \epsilon_{1}^{\prime}}{2}+\frac{\mu \epsilon_{2}^{\prime}}{2}+\sigma_{1}\left[\frac{-21}{16}+\frac{849 \epsilon_{1}^{\prime}}{32}-\frac{25 \mu}{8}-\right.$

$\left.\frac{801 \mu \epsilon_{1}^{\prime}}{32}+\frac{3}{2 \mu}-\frac{3 \epsilon_{1}^{\prime}}{2 \mu}\right]+\sigma_{2}\left[\frac{33}{16}-\frac{837 \epsilon_{1}^{\prime}}{32}+\frac{21 \mu}{16}-+\frac{837 \mu \epsilon_{1}^{\prime}}{32}-\frac{3}{2 \mu}+\right.$

$\left.\frac{3 \epsilon_{1}^{\prime}}{2 \mu}\right]+\frac{\sigma_{1}^{\prime}}{(1-\mu)}\left[\frac{-21}{8}-\frac{183 \mu}{16}+\frac{8113 \mu \epsilon_{2}^{\prime}}{144}+\frac{603 \mu^{2}}{32}-\frac{7789 \mu^{2} \epsilon_{2}^{\prime}}{144}\right]+$

$\frac{\sigma_{2}^{\prime}}{(1-\mu)}\left[\frac{9}{4}-\frac{507 \mu}{16}-\frac{279 \mu \epsilon_{2}^{\prime}}{16}+\frac{549 \mu^{2}}{32}+\frac{195 \mu^{2} \epsilon_{2}^{\prime}}{16}\right](2.14)$

Now transforming Eqs. (2.6) and (2.7) in matrix form, we get

$\mathrm{X}^{\prime}=\mathrm{PX}$

Where

$X=\left[\begin{array}{c}\xi \\ \eta \\ \xi^{\prime} \\ \eta^{\prime}\end{array}\right], X^{\prime}=\left[\begin{array}{c}\xi^{\prime} \\ \eta^{\prime} \\ \xi^{\prime \prime} \\ \eta^{\prime \prime}\end{array}\right]$

$\mathrm{P}(v, \mathrm{e})=\left[\begin{array}{cccc}0 & 0 & 1 & 0 \\ 0 & 0 & 0 & 1 \\ \varnothing \Omega_{\mathrm{xx}}^{0} & \varnothing \Omega_{\mathrm{xy}}^{0} & 0 & 2 \\ \varnothing \Omega_{\mathrm{yx}}^{0} & \varnothing \Omega_{\mathrm{yy}}^{0} & -2 & 0\end{array}\right]$

\section{Determination of characteristic exponents}

The variational equations of motion of the system are solved by exploiting the Floquet's theory, which determines the characteristics exponents in the system with periodic coefficients.

The solution of the system is represented as

$X_{K}=Y_{k} e^{\lambda_{k} v}$

Where $Y_{k}$ is periodic with period $T=2 \pi$ and $\lambda_{k}$ is the characteristic exponent of (2.16). Now dropping the suffix in (3.1), we get

$X=Y e^{\lambda v}$

Differentiating (3.2) w.r.t " $v "$ and substituting this value in (2.15), the variational equation takes the form

$\mathrm{Y}^{\prime}=(\mathrm{P}-\lambda \mathrm{I}) \mathrm{Y}$

Where I is the unit matrix of the same order as that of P.

Now using the expansion,

$\mathrm{Y}=\mathrm{Y}^{(0)}+\mathrm{eY}^{(1)}+\mathrm{e}^{2} \mathrm{Y}^{(2)}+\cdots$

$\lambda=\lambda_{0}+\mathrm{e} \lambda_{1}+\mathrm{e}^{2} \lambda_{2}+\cdots$

The matrix $\mathrm{P}$ is expanded as 
Where

$\mathrm{P}^{(0)}=\left[\begin{array}{cccc}0 & 0 & 1 & 0 \\ 0 & 0 & 0 & 1 \\ \Omega_{\mathrm{xx}}^{0} & \Omega_{\mathrm{xy}}^{0} & 0 & 2 \\ \Omega_{\mathrm{yx}}^{0} & \Omega_{\mathrm{yy}}^{0} & -2 & 0\end{array}\right]$

And

$\mathrm{P}^{(\mathrm{m})}=(-\cos v)^{\mathrm{m}} \mathrm{C}, \mathrm{m}=1,2,3, \ldots \ldots$

With

$\mathrm{C}=\left[\begin{array}{cccc}0 & 0 & 0 & 0 \\ 0 & 0 & 0 & 0 \\ \Omega_{\mathrm{xx}}^{0} & \Omega_{\mathrm{xy}}^{0} & 0 & 0 \\ \Omega_{\mathrm{yx}}^{0} & \Omega_{\mathrm{yy}}^{0} & 0 & 0\end{array}\right]$

Substituting the values of $\mathrm{Y}, \mathrm{Y}^{\prime}$ and $\mathrm{P}$ from (3.4) and (3.5) in (3.3), we get

$\mathrm{Y}^{\prime(0)}+\mathrm{eY}^{\prime(1)}+\mathrm{e}^{2} \mathrm{Y}^{\prime(2)}+\cdots=\left[\left\{\left(\mathrm{P}^{(0)}+\mathrm{eP}^{(1)}+\mathrm{e}^{2} \mathrm{P}^{(2)}+\cdots\right)-\right.\right.$

$\left.\mathrm{I}\left(\lambda_{0}+\mathrm{e} \lambda_{1}+\mathrm{e}^{2} \lambda_{2}+\cdots\right)\left(\mathrm{Y}^{(0)}+\mathrm{eY}^{(1)}+\mathrm{e}^{2} \mathrm{Y}^{(2)}+\cdots\right\}\right]$

Equating coefficient of terms with the same powers in e from both sides and using (3.4), we get

$\mathrm{Y}^{\prime(0)}+\left[\mathrm{I} \lambda_{0}-\mathrm{P}^{(0)}\right]=0$

$\mathrm{y}^{\prime(1)}+\left[\mathrm{I} \lambda_{0}-\mathrm{P}^{(0)}\right] \mathrm{Y}^{(1)}=\left(-\mathrm{C} \cos \mathrm{v}-\mathrm{I} \lambda_{1}\right) \mathrm{Y}^{(0)}$

$\mathrm{y}^{\prime(2)}+\left[\mathrm{I} \lambda_{0}-\mathrm{P}^{(0)}\right] \mathrm{Y}^{(2)}=\left(-\mathrm{C} \cos v-\mathrm{I} \lambda_{1}\right) \mathrm{Y}^{(1)}+\left(\mathrm{C} \cos ^{2} v-\right.$ $\left.\mathrm{I} \lambda_{2}\right) \mathrm{Y}^{(0)}$

$\mathrm{y}^{\prime(3)}+\left[\mathrm{I} \lambda_{0}-\mathrm{P}^{(0)}\right] \mathrm{Y}^{(3)}=\left(-\mathrm{C} \cos v-\mathrm{I} \lambda_{1}\right) \mathrm{Y}^{(2)}+\left(\mathrm{C} \cos ^{2} \mathrm{v}-\right.$ $\left.\mathrm{I} \lambda_{2}\right) \mathrm{Y}^{(1)}+\left(-\mathrm{C} \cos ^{3} v-\mathrm{I} \lambda_{3}\right) \mathrm{Y}^{(0)}$

Proceeding further, we obtain

$\mathrm{y}^{\prime(\mathrm{n})}+\left[\mathrm{I} \lambda_{0}-\mathrm{P}^{(0)}\right] \mathrm{Y}^{(\mathrm{n})}=\sum_{\mathrm{m}=1}^{\mathrm{n}}\left[\mathrm{C}(-\cos \mathrm{v})^{\mathrm{m}}-\mathrm{I} \lambda_{\mathrm{m}}\right] \mathrm{Y}^{(\mathrm{n}-\mathrm{m})}$

Assuming the constant vector for zeroth order solution, the nth order equation with non-homogeneous terms has frequencies up to $\mathrm{n} / 2 \pi$, including it. Let the particular solution be of the form

$\mathrm{Y}^{(\mathrm{n})}=\sum_{\mathrm{k}=-\mathrm{n}}^{\mathrm{k}=\mathrm{n}} \mathrm{a}^{(\mathrm{n}, \mathrm{k})} \mathrm{e}^{\mathrm{ik} v}, \mathrm{n}=0,1,2, \ldots$

Where

$a^{(n, k)}=\left[\begin{array}{c}a_{1}^{(n, k)} \\ a_{2}^{(n, k)} \\ a_{3}^{(n, k)} \\ a_{4}^{(n, k)}\end{array}\right]$

From Equations. (3.11) and (3.10), we obtain a system of equation which is required to determine $\lambda$ up to the $O\left(\mathrm{e}^{2}\right)$ as

$$
\begin{aligned}
& {\left[\mathrm{I} \lambda_{0}-\mathrm{P}^{(0)}\right] \mathrm{a}^{(0,0)}=0} \\
& {\left[\mathrm{I} \lambda_{0}-\mathrm{P}^{(0)}\right] \mathrm{a}^{(1,0)}=\lambda_{1} \mathrm{a}^{(0,0)}} \\
& {\left[\mathrm{I}\left(\lambda_{0}+\mathrm{i}\right)-\mathrm{P}^{(0)}\right] \mathrm{a}^{(1,1)}=-\frac{1}{2} \mathrm{C} \mathrm{a}^{(0,0)}}
\end{aligned}
$$

$\left[\mathrm{I}\left(\lambda_{0}-\mathrm{i}\right)-\mathrm{P}^{(0)}\right] \mathrm{a}^{(1,-1)}=-\frac{1}{2} \mathrm{C} \mathrm{a}^{(0,0)}$

$\left[\mathrm{I} \lambda_{0}-\mathrm{P}^{(0)}\right] \mathrm{a}^{(2,0)}=-\lambda_{1} \mathrm{a}^{(1,0)}+\left[\frac{-\mathrm{C}}{2}-\mathrm{I} \lambda_{2}\right] \mathrm{a}^{(0,0)}-$

$\frac{\mathrm{C}}{2}\left(\mathrm{a}^{(1,-1)}+\mathrm{a}^{(1,1)}\right)$

Now for existence of $\mathrm{a}^{(0,0)}$, it is clear from Eq. (3.13) that the required condition is

$\operatorname{det}\left(\mathrm{I} \lambda_{0}-\mathrm{P}^{(0)}\right)=0$

i.e $\left|\begin{array}{cccc}\lambda_{0} & 0 & -1 & 0 \\ 0 & \lambda_{0} & 0 & -1 \\ -\Omega_{\mathrm{xx}}^{0} & -\Omega_{\mathrm{xy}}^{0} & \lambda_{0} & -2 \\ -\Omega_{\mathrm{yx}}^{0} & -\Omega_{\mathrm{yy}}^{0} & 2 & \lambda_{0}\end{array}\right|=0$

On solving we get

$\lambda_{0}^{4}+\left(4-\Omega_{\mathrm{xx}}^{0}-\Omega_{\mathrm{yy}}^{0}\right) \lambda_{0}^{2}+\Omega_{\mathrm{xx}}^{0} \Omega_{\mathrm{yy}}^{0}-\left(\Omega_{\mathrm{xy}}^{0}\right)^{2}=0$

Or $\lambda_{0}^{4}-\mathrm{Q} \lambda_{0}^{2}+\mathrm{R}=0$

Where

$\mathrm{Q}=-\left(4-\Omega_{\mathrm{xx}}^{0}-\Omega_{\mathrm{yy}}^{0}\right), \mathrm{R}=\Omega_{\mathrm{xx}}^{0} \Omega_{\mathrm{yy}}^{0}-\left(\Omega_{\mathrm{xy}}^{0}\right)^{2}$

From Eq. (3.19) we have

$\lambda_{0}^{2}=\frac{\mathrm{Q} \pm \sqrt{\mathrm{Q}^{2}-4 \mathrm{R}}}{2}$

Substituting the values from Equations $(2.12,2.13 \& 2.14)$ into Equations. (3.20) and (3.21) we get

$\mathrm{Q}=-1+\frac{\epsilon_{1}^{\prime}}{2}-\frac{\epsilon_{2}^{\prime}}{2}-3 \mu \epsilon_{1}^{\prime}-\mu \epsilon_{2}^{\prime}+\sigma_{1}\left(\frac{-27}{16}+\frac{19 \mu}{16}+\frac{509 \epsilon_{1}^{\prime}}{16}-\right.$

$\left.\frac{105 \mu \epsilon_{1}^{\prime}}{4}+\frac{3}{4 \mu}+\frac{\epsilon_{1}^{\prime}}{4 \mu}\right)+\sigma_{2}\left(\frac{63}{16}-\frac{27 \mu}{8}-\frac{731 \epsilon_{1}^{\prime}}{16}+\frac{359 \mu \epsilon_{1}^{\prime}}{8}-\frac{3}{4 \mu}-\frac{\epsilon_{1}^{\prime}}{4 \mu}\right)+$

$\sigma_{1}^{\prime}\left(\frac{33}{16}-\frac{147 \mu}{32}+\frac{3 \epsilon_{2}^{\prime}}{16}+\frac{191 \mu \epsilon_{2}^{\prime}}{16}+\frac{621 \mu^{2}}{32(1-\mu)}-\frac{1981 \mu^{2} \epsilon_{2}^{\prime}}{36(1-\mu)}-\frac{21}{8(1-\mu)}-\right.$

$\left.\frac{183 \mu}{16(1-\mu)}+\frac{8113 \mu \epsilon_{2}^{\prime}}{144(1-\mu)}\right)+\sigma_{2}^{\prime}\left(\frac{-33}{16}+\frac{207 \mu}{32}-\frac{7 \epsilon_{2}^{\prime}}{16}-\frac{483 \mu \epsilon_{2}^{\prime}}{32}+\frac{507 \mu^{2}}{32(1-\mu)}+\right.$

$\left.\frac{115 \mu^{2} \epsilon_{2}^{\prime}}{8(1-\mu)}+\frac{9}{4(1-\mu)}-\frac{507 \mu}{16(1-\mu)}-\frac{279 \mu \epsilon_{2}^{\prime}}{16(1-\mu)}\right)$

$\mathrm{R}=\frac{9 \epsilon_{1}^{\prime}}{8}-\frac{9 \epsilon_{2}^{\prime}}{8}+\frac{27 \mu}{4}-\frac{27 \mu^{2}}{4}-\frac{45 \mu \epsilon_{1}^{\prime}}{8}+\frac{21 \mu \epsilon_{2}^{\prime}}{2}-\frac{27 \mu^{2} \epsilon_{1}^{\prime}}{36}-21 \mu^{2} \epsilon_{2}^{\prime}+$ $\sigma_{1}\left(\frac{-603}{64}+\frac{1593 \epsilon_{1}^{\prime}}{64}+\frac{1173 \mu}{64}+\frac{471 \mu \epsilon_{1}^{\prime}}{64}+\frac{27}{16 \mu}-\frac{71 \epsilon_{1}^{\prime}}{16 \mu}-\frac{689 \mu^{2} \epsilon_{1}^{\prime}}{16}-\right.$ $\left.\frac{153 \mu^{2}}{16}\right)+\sigma_{2}\left(\frac{495}{64}-\frac{3619 \epsilon_{1}^{\prime}}{64}-\frac{639 \mu}{64}-18 \mu \epsilon_{1}^{\prime}-\frac{27}{16 \mu}+\frac{71 \epsilon_{1}^{\prime}}{16 \mu}+\right.$

$\left.\frac{1455 \mu^{2} \epsilon_{1}^{\prime}}{32}+\frac{351 \mu^{2}}{32}\right)+\sigma_{1}^{\prime}\left(\frac{45}{64}+\frac{287 \epsilon_{2}^{\prime}}{64}-\frac{873 \mu}{128}+\frac{3663 \mu \epsilon_{2}^{\prime}}{64}-\frac{3143 \mu^{2} \epsilon_{2}^{\prime}}{64}+\right.$ $\frac{2007 \mu^{2}}{128(1-\mu)}+\frac{279 \mu^{2}}{32}-\frac{63}{32(1-\mu)}-\frac{549 \mu}{64(1-\mu)}-\frac{21 \epsilon_{2}^{\prime}}{16(1-\mu)}+\frac{7771 \mu \epsilon_{2}^{\prime}}{192(1-\mu)}-$

$\left.\frac{2975 \mu^{2} \epsilon_{2}^{\prime}}{192(1-\mu)}\right)+\sigma_{2}^{\prime}\left(\frac{-1107}{16}-\frac{69 \epsilon_{2}^{\prime}}{64}+\frac{2205 \mu}{128}-\frac{13399 \mu \epsilon_{2}^{\prime}}{128}+\frac{5591 \mu^{2} \epsilon_{2}^{\prime}}{64}-\right.$

$\frac{117 \mu^{2}}{256(1-\mu)}-\frac{819 \mu^{2}}{32}+\frac{27}{16(1-\mu)}-\frac{1521 \mu}{64(1-\mu)}+\frac{9 \epsilon_{2}^{\prime}}{32(1-\mu)}-\frac{615 \mu \epsilon_{2}^{\prime}}{32(1-\mu)}+$

$\left.\frac{4379 \mu^{2} \epsilon_{2}^{\prime}}{64(1-\mu)}\right)$

From the first equation of the system of equation (3.13), we observe that it is necessary that the coefficient on the left with any column replaced by the non-homogeneous terms on the right must be zero

$\operatorname{det} \cdot\left[\mathrm{I} \lambda_{0}-\mathrm{P}^{(0)}\right]+\lambda_{1} \mathrm{a}^{(0,0)}=0$

Since $\lambda$ enters as a factor in all elements of the replaced column, hence

$\lambda_{1}$ det. $\left[\mathrm{I} \lambda_{0}-\mathrm{P}^{(0)}\right]+\mathrm{a}^{(0,0)}=0$ 
But

$\operatorname{det} .\left[\mathrm{I} \lambda_{0}-\mathrm{P}^{(0)}\right] \neq 0$

Therefore

$$
\lambda_{1}=0
$$

Again from Equations. (3.15) and (3.16), the solutions of $\mathrm{a}^{(1,1)}$ and $\mathrm{a}^{(1,-1)}$ are

$\mathrm{a}^{(1,1)}=-\frac{1}{2}\left[\mathrm{I}\left(\lambda_{0}+\mathrm{i}\right)-\mathrm{P}^{(0)}\right]^{-1} \mathrm{C} \mathrm{a}^{(0,0)}$

$\mathrm{a}^{(1,-1)}=-\frac{1}{2}\left[\mathrm{I}\left(\lambda_{0}-\mathrm{i}\right)-\mathrm{P}^{(0)}\right]^{-1} \mathrm{C} \mathrm{a}^{(0,0)}$

Substituting this value of $\mathrm{a}^{(1,1)}$ and $\mathrm{a}^{(1,-1)}$ in the last equation We get;

$\left[\mathrm{I} \lambda_{0}-\mathrm{P}^{(0)}\right] \mathrm{a}^{(2,0)}=\frac{\mathrm{C}}{4}\left[\left(\mathrm{I}\left(\lambda_{0}+\mathrm{i}\right)-\mathrm{P}^{(0)}\right)^{-1}+\left(\mathrm{I}\left(\lambda_{0}-\mathrm{i}\right)-\right.\right.$

$\left.\left.\mathrm{P}^{(0)}\right)^{-1}\right] \mathrm{C} \mathrm{\textrm {a } ^ { ( 0 , 0 ) }}+\left(\frac{\mathrm{C}}{2}-\mathrm{I} \lambda_{2}\right) \mathrm{a}^{(0,0)}$

The matrices within the square bracket are complex conjugate so considering real part only

$\left[\mathrm{I} \lambda_{0}-\mathrm{P}^{(0)}\right] \mathrm{a}^{(2,0)}=\left[\frac{\mathrm{C}}{2} \operatorname{Re}\left(\mathrm{I}\left(\lambda_{0}+\mathrm{i}\right)-\mathrm{P}^{(0)}\right)^{-1}+\left(\frac{\mathrm{C}}{2}-\right.\right.$

$\left.\left.\mathrm{I} \lambda_{2}\right)\right] \mathrm{a}^{(0,0)}$

After some mathematical manipulations, the value of $\lambda_{2}$ can be obtained from (3.29) given by

$$
\begin{aligned}
& \lambda_{2}=-\frac{\left(Q^{2}-4 R-16\right) \lambda_{0}^{2}+A_{0} F_{0}+A_{1} F_{1}+A_{2} F_{2}}{4\left(Q^{2}-4 Q-4 R\right) \lambda_{0}^{2}+32 R} \lambda_{0} \\
& \text { Or }
\end{aligned}
$$

$$
\lambda_{2}=\mathrm{A} \lambda_{0}
$$

Where

$$
\begin{aligned}
& A=-\frac{\left(Q^{2}-4 R-16\right) \lambda_{0}^{2}+A_{0} F_{0}+A_{1} F_{1}+A_{2} F_{2}}{4\left(Q^{2}-4 Q-4 R\right) \lambda_{0}^{2}+32 R} \\
& A_{0}=\left[(Q+4)^{2}(Q+4)-4 Q R\right] \lambda_{0}^{2}-R(Q+4)-4 R^{2} \\
& \mathrm{~F}_{0}=\frac{1}{\mathrm{~N}}\left[(\mathrm{Q}+1) \lambda_{0}^{2}+(\mathrm{Q}+1)+2 \mathrm{R}\right] \\
& A_{1}=-8 \lambda_{0} R\left[2 \lambda_{0}^{2}-(Q+4)\right] \\
& \mathrm{F}_{1}=-\frac{\lambda_{0}}{\mathrm{~N}}\left[2 \lambda_{0}^{2}+(\mathrm{Q}+3)\right] \\
& A_{2}=-8 \lambda_{0}^{2} R\left(Q^{2}-4 R-16\right) \\
& \mathrm{F}_{2}=-\frac{1}{\mathrm{~N}}\left[\lambda_{0}^{2}-(\mathrm{Q}+1)\right] \\
& \mathrm{F}_{1}=-\frac{\lambda_{0}}{\mathrm{~N}}\left[2 \lambda_{0}^{2}+(\mathrm{Q}+3)\right] \\
& N=\left[4 Q^{2}+8 Q+4-16 R\right] \lambda_{0}^{2}-4 R+(Q+1)^{2}
\end{aligned}
$$

Using value of $Q$ i.e

$\mathrm{Q}=-1+\frac{\epsilon_{1}^{\prime}}{2}-\frac{\epsilon_{2}^{\prime}}{2}-3 \mu \epsilon_{1}^{\prime}-\mu \epsilon_{2}^{\prime}+\sigma_{1}\left(\frac{-27}{16}+\frac{19 \mu}{16}+\frac{509 \epsilon_{1}^{\prime}}{16}-\right.$

$\left.\frac{105 \mu \epsilon_{1}^{\prime}}{4}+\frac{3}{4 \mu}+\frac{\epsilon_{1}^{\prime}}{4 \mu}\right)+\sigma_{2}\left(\frac{63}{16}-\frac{27 \mu}{8}-\frac{731 \epsilon_{1}^{\prime}}{16}+\frac{359 \mu \epsilon_{1}^{\prime}}{8}-\frac{3}{4 \mu}-\frac{\epsilon_{1}^{\prime}}{4 \mu}\right)+$ $\sigma_{1}^{\prime}\left(\frac{33}{16}-\frac{147 \mu}{32}+\frac{3 \epsilon_{2}^{\prime}}{16}+\frac{191 \mu \epsilon_{2}^{\prime}}{16}+\frac{621 \mu^{2}}{32(1-\mu)}-\frac{1981 \mu^{2} \epsilon_{2}^{\prime}}{36(1-\mu)}-\frac{21}{8(1-\mu)}-\right.$

$\left.\frac{183 \mu}{16(1-\mu)}+\frac{8113 \mu \epsilon_{2}^{\prime}}{144(1-\mu)}\right)+\sigma_{2}^{\prime}\left(\frac{-33}{16}+\frac{207 \mu}{32}-\frac{7 \epsilon_{2}^{\prime}}{16}-\frac{483 \mu \epsilon_{2}^{\prime}}{32}+\frac{507 \mu^{2}}{32(1-\mu)}+\right.$

$\left.\frac{115 \mu^{2} \epsilon_{2}^{\prime}}{8(1-\mu)}+\frac{9}{4(1-\mu)}-\frac{507 \mu}{16(1-\mu)}-\frac{279 \mu \epsilon_{2}^{\prime}}{16(1-\mu)}\right)$

We find the value of the parameter "A", which is calculated as mentioned below

$A=-\frac{E}{F}$

Where

$E=\left(Q^{2}-4 R-16\right) \lambda_{0}^{2}+A_{0} F_{0}+A_{1} F_{1}+A_{2} F_{2}$

And

$\mathrm{F}=4\left(\mathrm{Q}^{2}-4 \mathrm{Q}-4 \mathrm{R}\right) \lambda_{0}^{2}+32 \mathrm{R}$

Hence

$\lambda_{2}=\mathrm{A} \lambda_{0}$

Thus, the characteristic exponent up to second order of approximation in " $\mathrm{e}$ " can be written as

$\lambda=\lambda_{0}+\mathrm{e}^{2} \lambda_{2}$

\section{Transition curves separating stable and un- stable regions}

The transition curves describe the stability of the triangular equilibrium points in the elliptical restricted three body problem by separating stable and unstable regions. It can be found by simply equating the expression for the characteristic roots or the exponents to the value for periodic solution in the range of $0 \leq \mu \leq \frac{1}{2}$ From Floquet's theory, if $\lambda_{\mathrm{k}}$ are the characteristics exponents, then in polar form it can be written as

$\lambda_{\mathrm{k}}=\frac{1}{\mathrm{~T}}\left[\left(\operatorname{In} \mathrm{b}_{\mathrm{k}}+\mathrm{i}\left(\theta_{\mathrm{k}}+2 \mathrm{n} \pi\right)\right], \mathrm{n}=0, \pm 1, \pm 2\right.$.

The real part of the exponent determines whether the solution is bounded or not. So, the corresponding periodic solution from (4.1) is

$\lambda=\mathrm{i}\left(\mathrm{n} \pm \frac{1}{2}\right), \mathrm{n}=0, \pm 1, \pm 2$

In the range $0 \leq \mu \leq \frac{1}{2}$, the periodic solution provides

$\lambda^{*}= \pm \frac{\mathrm{i}}{2}$

Replacing $\lambda$ by $\lambda^{*}$ in (3.36) we obtain

$\pm \frac{\mathrm{i}}{2}=\left(1+\mathrm{e}^{2} \mathrm{~A}\right) \lambda_{0}$

After further simplification we obtain

$\mathrm{e}^{2}=\left[ \pm\left(-\frac{1}{4 \lambda_{0}^{2}}\right)^{\frac{1}{2}}-1\right] \frac{1}{\mathrm{~A}}$

\section{Conclusion and discussion}

The stability of the triangular equilibrium points in ER3BP under the effect of radiating primaries has been investigated using the analytical method developed by Bennet[3,4].this method is based 
on flouqet theory. Narayan and Usha [15] examined the stability of primaries under the assumption that the bigger primary is source of radiation and smaller one is a triaxial rigid body, and it has been seen that effect of radiation pressure is more significant than the oblateness and triaxiality.In another paper,Narayan and singh [16] investigated the linear stability of triangular points under the assumption that bothe the primaries are source of radiation. The Authors Narayan,A., Pandey, K.K and Shrivastava,S.K. [17] has been investigated the stability of system under the assumption that both the primaries are source of radiation and triaxial as well ,by Grebenikov method [7].a minor dissimilarity is noticed in the results ,which may be because of use of different Method.

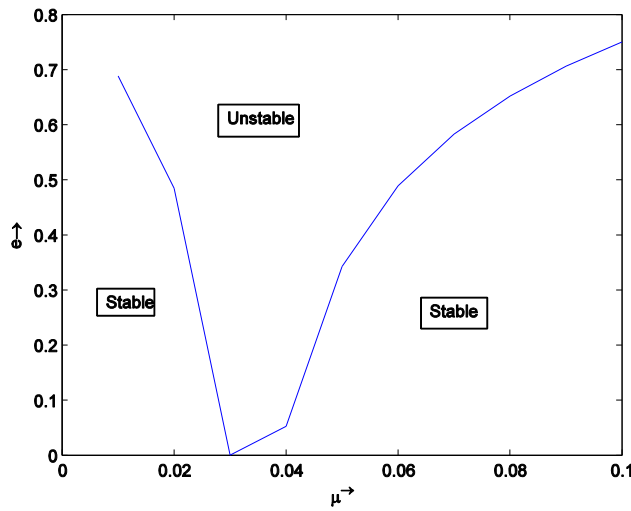

(Transition Curve 1: $\sigma_{1}, \sigma_{2}, \sigma_{1}^{\prime}, \sigma_{2}^{\prime}, \epsilon_{1}^{\prime}, \epsilon_{2}^{\prime}=0$ )

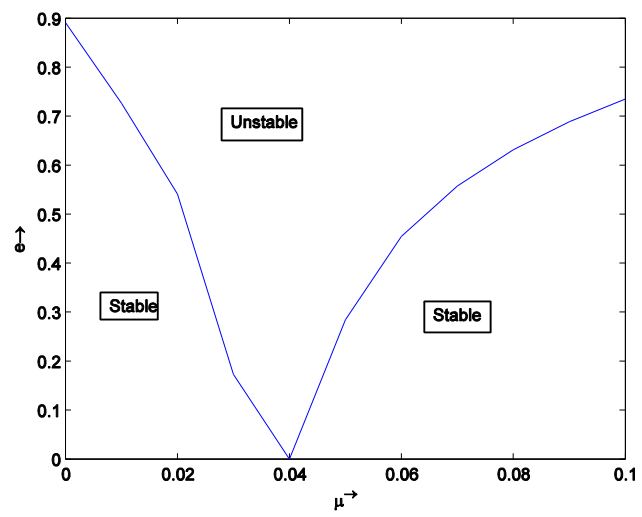

(Transition Curve 2: $\sigma_{1}=0.0003, \sigma_{2}=0.0002, \sigma_{1}^{\prime}=$ $\left.0.0001, \sigma_{2}^{\prime=} 0.0002, \epsilon_{1}^{\prime}=0.001, \epsilon_{2}^{\prime}=0.002\right)$

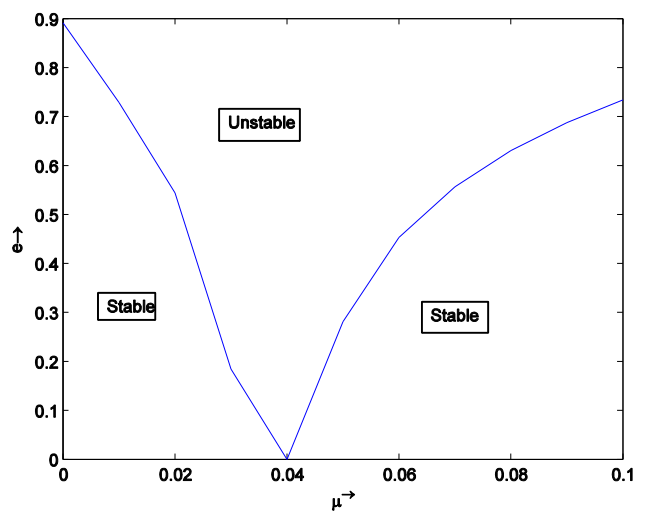

(Transition Curve 3: $\sigma_{1}=0.0004, \sigma_{2}=0.0002, \sigma_{1}^{\prime}=$ $0.0001, \sigma_{2}^{\prime}=0.0002, \epsilon_{1}^{\prime}=0.001, \epsilon_{2}^{\prime}=0.002$ )

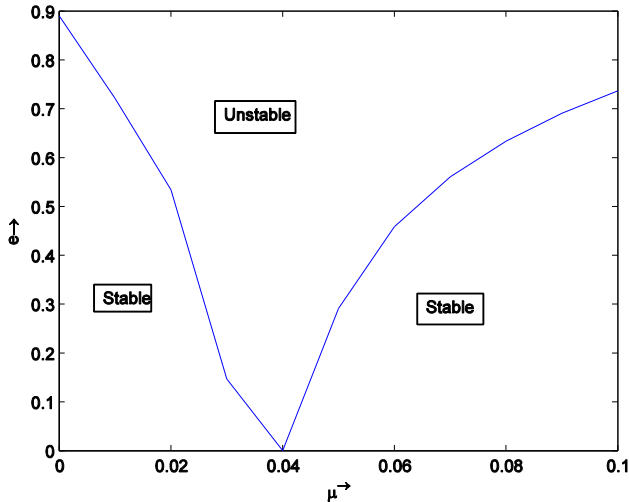

(Transition Curve 4: $\sigma_{1}=0.0001, \sigma_{2}=0.0002, \sigma_{1}^{\prime}=$ $\left.0.0001, \sigma_{2}^{\prime}=0.0002, \epsilon_{1}^{\prime}=0.001, \epsilon_{2}^{\prime}=0.002\right)$.

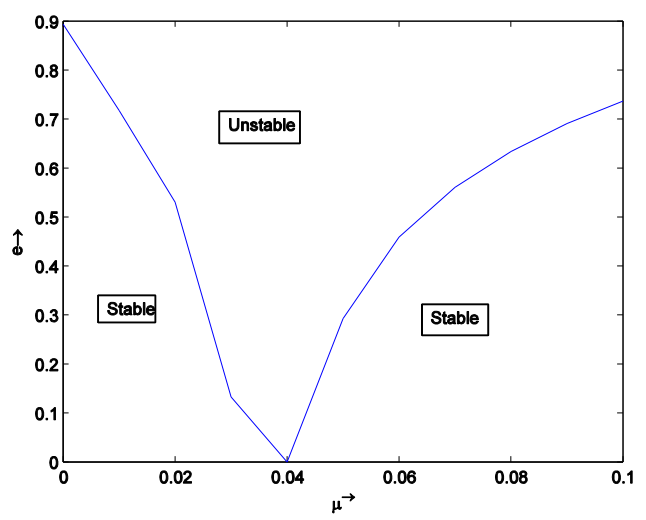

(Transition Curve 5: $\sigma_{1}=0.0001, \sigma_{2}=0.0003, \sigma_{1}^{\prime}=$ $0.0001, \sigma_{2}^{\prime}=0.0003, \epsilon_{1}^{\prime}=0.001, \epsilon_{2}^{\prime}=0.002$ ).

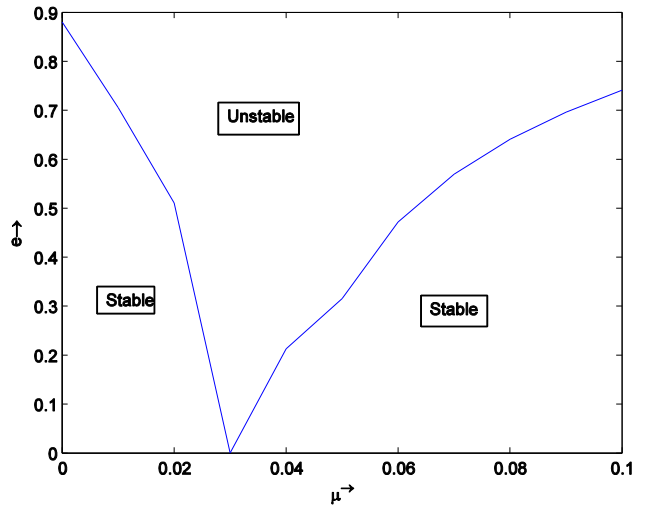

(Transition Curve 6: $\sigma_{1}=0.0001, \sigma_{2}=0.0002, \sigma_{1}^{\prime}=$ $0.0002, \sigma_{2}^{\prime}=0.0001, \epsilon_{1}^{\prime}=0.001, \epsilon_{2}^{\prime}=0.002$ ).

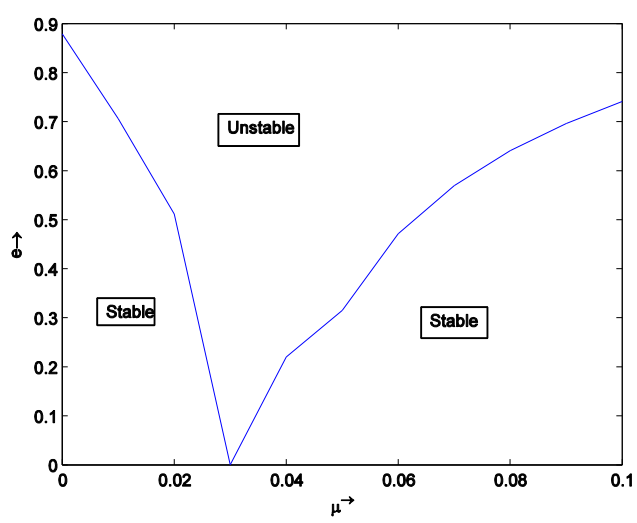

(Transition Curve 7: $\sigma_{1}=0.0001, \sigma_{2}=0.0002, \sigma_{1}^{\prime}=$ $\left.0.0003, \sigma_{2}^{\prime}=0.0001, \epsilon_{1}^{\prime}=0.001, \epsilon_{2}^{\prime}=0.002\right)$. 


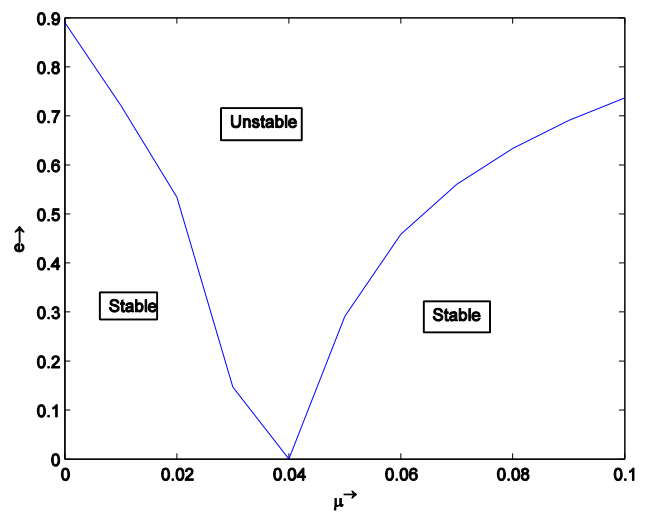

(Transition Curve 8: $\sigma_{1}=0.0001, \sigma_{2}=0.0002, \sigma_{1}^{\prime}=$ $0.0001, \sigma_{2}^{\prime}=0.0002, \epsilon_{1}^{\prime}=0.001, \epsilon_{2}^{\prime}=0.002$ ).

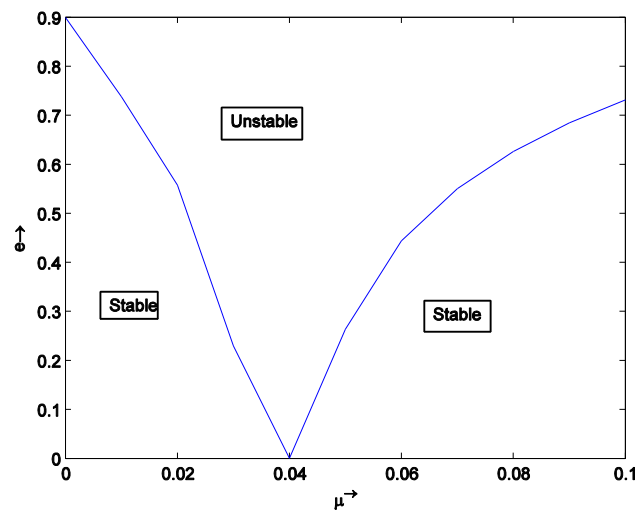

(Transition Curve 9: $\sigma_{1}=0.0001, \sigma_{2}=0.0002, \sigma_{1}^{\prime}=$ $0.0001, \sigma_{2}^{\prime}=0.0003, \epsilon_{1}^{\prime}=0.001, \epsilon_{2}^{\prime}=0.002$ ).

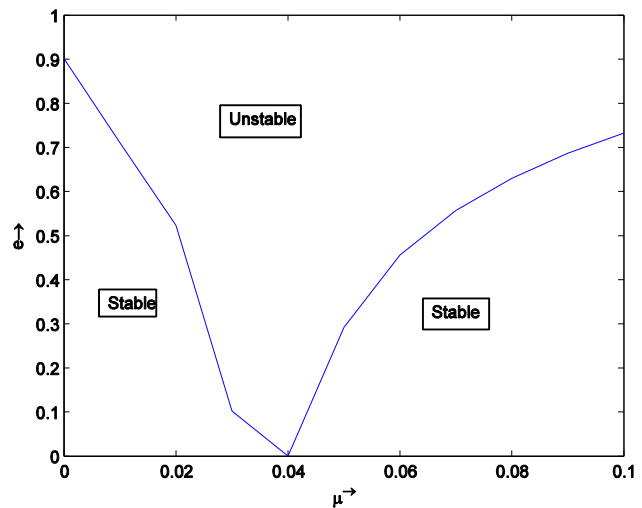

(Transition Curve 10: $\sigma_{1}=0.0001, \sigma_{2}=0.0002, \sigma_{1}^{\prime}=$ $0.0001, \sigma_{2}^{\prime}=0.0002, \epsilon_{1}^{\prime}=0.002, \epsilon_{2}^{\prime}=0.001$ ).

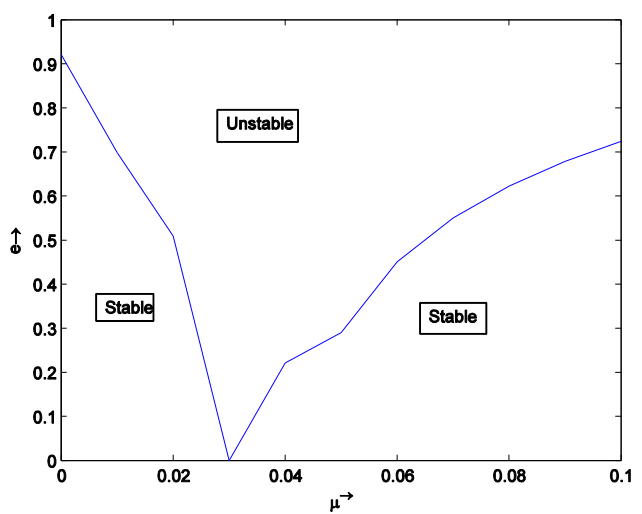

(Transition Curve 11: $\sigma_{1}=0.0001, \sigma_{2}=0.0002, \sigma_{1}^{\prime}=$ $\left.0.0001, \sigma_{2}^{\prime}=0.0002, \epsilon_{1}^{\prime}=0.004, \epsilon_{2}^{\prime}=0.001\right)$.

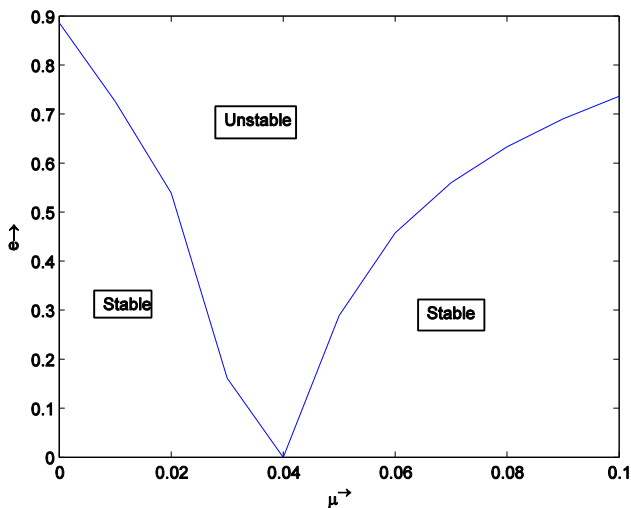

(Transition Curve 12: $\sigma_{1}=0.0001, \sigma_{2}=0.0002, \sigma_{1}^{\prime}=$ $0.0001, \sigma_{2}^{\prime}=0.0002, \epsilon_{1}^{\prime}=0.001, \epsilon_{2}^{\prime}=0.003$ ).

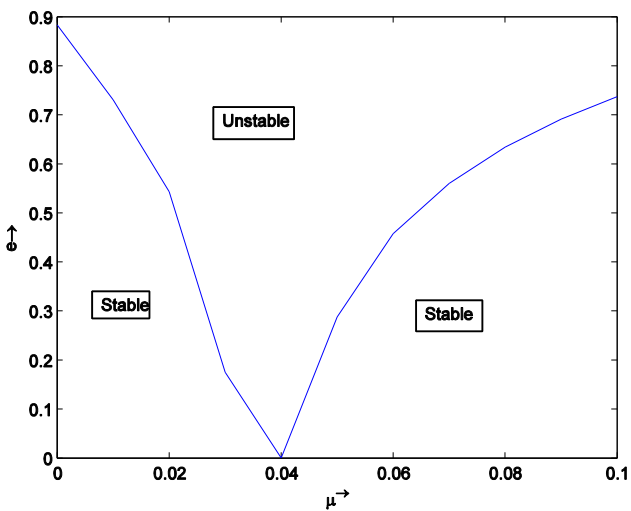

(Transition Curve 13: $\sigma_{1}=0.0001, \sigma_{2}=0.0002, \sigma_{1}^{\prime}=$ $\left.0.0001, \sigma_{2}^{\prime}=0.0002, \epsilon_{1}^{\prime}=0.001, \epsilon_{2}^{\prime}=0.004\right)$.

\section{References}

[1] Ammar, M.K., The effect of solar radiation pressure on the Lagrangian points in the elliptical restricted three body problem, Astrophysics and Space Science, 313 , 393408(2008)https://doi.org/10.1007/s10509-007-9709-z.

[2] Alfriend, K.T., Rand, R. H.: Stability of the triangular points in the elliptic restricted problem of three bodies. AIAAJ 6, 1024(1969)https://doi.org/10.2514/3.5270.

[3] Bennet, A.: In Astrodynamicssecialist Conference. Monetary, California. 101 (1965)

[4] Bennet, A.: Characteristic exponents of the five equilibrium solutions in the Elliptically Restricted Problem, ICARUS 4, 177 187(1965)https://doi.org/10.1016/0019-1035(65)90060-6.

[5] Danby JMA.: Stability of the triangular points in the elliptical restricted problem of three bodies, Astronomical Journal, 69,165-172 (1964)https://doi.org/10.1086/109254.

[6] Deprit, A., Rom, A.: Characteristics exponents at L4 in the elliptic restricted problem. Astron. Astrphysc.5, 416-425(1970)

[7] Grebnikov,E.A., On the stability of the Lagrangian triangle solutions of the restricted elliptic three body problem, Soviet Astronomy, 8 , No.3, 567-578(1964)

[8] Gyorgyey ,J, On the non-linear motions around the elliptical restricted problem of three bodies, Celestial Mechanics and Dynamical Astronomy, 36, No.3, 281285(1985)https://doi.org/10.1007/BF01230741.

[9] Kumar, S., Ishwar B.: Location of collinear equilibrium points in the generalized photogravitational elliptic restricted three body $\begin{array}{lllll}\text { problem. Int.J.Eng. Sci. Tech.3 (2), 157-162 } & \end{array}$ (2011)https://doi.org/10.4314/ijest.v3i2.68143.

[10] Kumar, V., Choudhary , R. K.,Non-linear stability of the triangular libration points for the photogravitational elliptic restricted problem of three bodies, Celestial Mechanics and Dynamical Astronomy,48, No.4, 299-317(1990)https://doi.org/10.1007/BF00049387.

[11] Markellos, V. V., Perdios, E., Labrapoulou, P., Linear stability of the triangular equilibrium points in the photogravitational elliptic restricted three body problem, Astrophysics and Space Science ,194, 207-214(1992)https://doi.org/10.1007/BF00643991.

[12] Moulton, F.R.: On the stability of direct and retrograde orbits. Astron. Soc. 75, 40-57 (1914)https://doi.org/10.1093/mnras/75.2.40. 
[13] Narayan, A., Kumar, C.R. Effects of photogravitational and oblateness on the Triangular Lagrangian points in the Elliptical Restricted three body problem, Indian Journal of Pure and Applied Mathematics,68, No.2, 201-224(2011)

[14] Narayan, A., Singh, N.: Motion and stability of triangular equilibrium points in elliptical restricted three body problem under the radiating primaries. Astrophys. Space Sci.352 (1), 57-70 (2014)https://doi.org/10.1007/s10509-014-1903-1.

[15] Narayan, A., Usha, T., Stability of triangular equilibrium points in the elliptic restricted problem of three bodies with radiation and triaxial primaries, Astrophysics and Space Science,351(1), 135142(2014)https://doi.org/10.1007/s10509-014-1818-x.

[16] Narayan,A., Singh, N.: Stability of triangular points in elliptical restricted three body problem under the radiating binary system . Astrophys. Space Sci. doi: 10.1007/s 10509-014-2014-8

[17] Narayan,A., Pandey, K.K and Shrivastava,S.K.: Effects of radiation and triaxiality of triangular equilibrium points in elliptical restricted three body problem. International Journal of Advanced Astronomy 3(2), 97-106 (2015).https://doi.org/10.14419/ijaa.v3i2.5302.

[18] Singh, J., Aishetu, U., Motion in the photogravitational elliptic restricted three body problem under an oblate primary, Astronomical Journal, 143, No.5, 109(22pp)(2012)

[19] Singh, J., Aishetu, U., On the stability of triangular points in the elliptic restricted three bodies under the radiating and oblate Primaries, Astrophysics and Space Science ,341, 349358(2012)https://doi.org/10.1007/s10509-012-1109-3.

[20] Szebebely, V.: Stability of the points of equilibrium in the restricted problem. Astron.J. 72,7-9(1967)https://doi.org/10.1086/110195.

[21] Usha, T., Narayan, A., Ishwar, B., Effects of radiation and triaxiality of primaries on triangular equilibrium points in elliptic restricted three body problem, Astrophysics and Space Science,349(1), 151164(2014)https://doi.org/10.1007/s10509-013-1655-3.

[22] Zimvoschikov, A.S., Thkai, V.N., Instability of libration points and resonance phenomena in the photogravitational elliptical restricted three body problem, Solar system research, 38, No.2, 155 163(2004).https://doi.org/10.1023/B:SOLS.0000022826.31475.a7. 